\title{
Pregnancy with Suspected Pulmonary Arterial Hypertension: A Referral for Confirmation Prior to Termination of Pregnancy
}

\author{
Cody Schwartz ${ }^{1}$, Neeraj Jain ${ }^{1},{\text { Murtuza } \text { Ali }^{1} \text {, and Viviana Falco }}^{1}$ \\ ${ }^{1}$ Louisiana State University Health Sciences Center
}

February 12, 2021

\begin{abstract}
Certain cardiovascular conditions pose a significant maternal-fetal risk during pregnancy with the greatest risk seen in pulmonary arterial hypertension (PAH). Current guidelines clearly recommend the avoidance of pregnancy in women with PAH and termination when pregnancy does occur but only after the diagnosis of PAH has been confirmed by right heart catheterization (RHC).
\end{abstract}

Title Page

Title: Pregnancy with Suspected Pulmonary Arterial Hypertension: A Referral for Confirmation Prior to Termination of Pregnancy

Running Head: Confirming PAH Prior to Termination of Pregnancy

Authors: Cody Schwartz, DO ${ }^{1}$; Neeraj Jain, $\mathrm{MD}^{1}$; Murtuza Ali, $\mathrm{MD}^{1}$; Viviana Falco, $\mathrm{MD}^{1}$

Department/Institution: ${ }^{1}$ Department of Cardiology, LSU Health-New Orleans School of Medicine, New Orleans, LA

\section{Corresponding Author:}

Cody Schwartz, DO

533 Bolivar St

Room 360

New Orleans, LA 70112

P: 813-240-0488

F: 504-568-2127

E: cschw9@lsuhsc.edu

Grants: None

Abstract:

Certain cardiovascular conditions pose a significant maternal-fetal risk during pregnancy with the greatest risk seen in pulmonary arterial hypertension $(\mathrm{PAH})$. Current guidelines clearly recommend the avoidance of pregnancy in women with PAH and termination when pregnancy does occur but only after the diagnosis of $\mathrm{PAH}$ has been confirmed by right heart catheterization (RHC).

Key Words: 
Pulmonary Artery Hypertension

Right Ventricular Function

Mid-cavitary Obstruction

Transthoracic Echocardiography

Congenital Heart Defects

\section{Case:}

A 37-year-old pregnant female with a past medical history notable for a murmur as a child presented for a right heart catheterization (RHC) to confirm the diagnosis of suspected pulmonary arterial hypertension (PAH) discovered on a transthoracic echocardiogram (TTE). The results of the TTE showed a normal left ventricular systolic function and ejection fraction however there was right-sided chamber enlargement with a right ventricular (RV) systolic pressure of $74 \mathrm{mmHg}$ calculated from a max tricuspid regurgitant pressure gradient of $66 \mathrm{mmHg}$ and a right atrial pressure of $8 \mathrm{mmHg}$. Maternal-Fetal Medicine was considering terminating the pregnancy but wanted confirmation of PAH prior to making any final decisions. Her physical exam findings were negative for a murmur but did show tachycardia. She had an electrocardiogram that showed sinus tachycardia but was otherwise unremarkable. Hemodynamic results of the RHC showed a right atrial pressure of $3 \mathrm{mmHg}$, a RVbase pressure of $53 / 3 \mathrm{mmHg}$, a RV outflow tract pressure of $27 / 3 \mathrm{mmHg}$, a pulmonary artery pressure of $27 / 7 \mathrm{mmHg}$, and a pulmonary capillary wedge pressure of $7 \mathrm{mmHg}$ (Figure 1).

The results of the RHC were unexpected as there was no evidence of PAH but, instead, showed a significant RV intracavitary gradient. Given the RHC findings, we decided to perform a more focused TTE to investigate the etiology of the RV intracavitary gradient. Results of the TTE showed a prominent RV moderator band producing a double-chambered RV with no evidence of a ventricular septal defect (VSD) and hemodynamics congruent with those found on the RHC (Figure 2).

From a cardiac standpoint, given the results of the RHC and the repeat TTE, it was deemed appropriate for the patient to proceed with the pregnancy but under close clinical monitoring.

\section{Discussion:}

Cardiac disease in the pregnant patient can present challenges in cardiovascular and maternal-fetal management. Even in patients with no history of cardiopulmonary disease, pregnancy imposes some dramatic physiologic changes upon the cardiovascular system. These changes include an increase in plasma volume by $50 \%$, an increase in resting pulse by $17 \%$, a decrease in systemic vascular resistance by $20 \%$ and an increase in cardiac output by $50 \%$. After delivery, the heart rate normalizes within 10 days; by 3 months postpartum, stroke volume, cardiac output, and systemic vascular resistance return to the pre-pregnancy state.

In women with certain preexisting cardiovascular conditions, pregnancy may be contraindicated, and they should be advised to avoid pregnancy. These conditions include significant PAH of any cause, severe mitral stenosis, severe symptomatic aortic stenosis, a bicuspid aortic valve with an aorta diameter $>50 \mathrm{~mm}$, Marfan syndrome with a dilated aorta $>45 \mathrm{~mm}$, severe systemic ventricular systolic dysfunction (left ventricular ejection fraction $<30$ percent, New York Heart Association III to IV), and native severe coarctation. Among the conditions deemed high risk cardiac pregnancy states, PAH confers the highest risk to the patient and/or the fetus. PAH is a histopathological disorder characterized by an increase in blood pressure within arteries and/or capillaries of the lungs, leading to heart failure, debilitating symptoms and shortened life expectancy. PAH in pregnancy confers a combined maternal and fetal mortality rate that approaches $50 \%$. Management of PAH in pregnancy generally consists of terminating the pregnancy. On the other hand, a double-chambered RV is a rare heart defect in which the RV is separated into a high-pressure proximal and low-pressure distal chamber. This defect is considered to be congenital and typically presents in infancy or childhood but has been reported to present rarely in adults. Usually, the obstruction is caused by an anomalous muscle bundle (moderator band) crossing the RV from the interventricular septum to the RV 
free wall. The most commonly associated cardiac anomaly with this condition is a VSD and can be seen in up to $90 \%$ patients with a double-chambered RV. It is important to note, an increased tricuspid regurgitant gradient, as measured by continuous-wave Doppler on echocardiogram, may be misdiagnosed as pulmonary hypertension instead of a double-chambered RV, as was seen in our case. Surgery is the primary treatment option for a double-chambered RV and should be offered to patients who are symptomatic and who have an elevated pressure gradient greater than $40 \mathrm{mmHg}$.

\section{Conclusion:}

It is important to recognize that estimated pulmonary artery pressures on TTE may be deemed as falsely elevated due routine equating of RV and pulmonary artery systolic pressures especially in this case of doublechambered RV physiology. Consequently, this miscalculation may lead to the incorrect diagnosis of PAH. While a TTE is a useful tool, RHC is the most confident method to diagnose PAH. Therapy should not be initiated based on pulmonary artery pressure results from TTE, especially in pregnancy when termination is being considered.

\section{Learning Objectives:}

- Pulmonary arterial hypertension is a cardiovascular condition that poses the highest maternal-fetal risk during pregnancy therefore making it a contraindication to pregnancy

- When pregnancy does occur in a patient with pulmonary arterial hypertension, termination of pregnancy is often required, however, prior to termination, the diagnosis of pulmonary arterial hypertension must be confirmed by right heart catheterization

- Right heart catheterization is currently the only way to officially diagnose pulmonary arterial hypertension

- While the echocardiogram is a useful tool, it cannot be used to officially diagnose pulmonary arterial hypertension and treatment should not be initiated based on estimated pulmonary artery pressures from the echocardiogram

- It is important to recognize that estimated pulmonary artery pressures on echocardiogram may be falsely elevated due to a prominent moderator band in the right ventricle leading to a double-chambered right ventricle with a significant intracavitary gradient

\section{Authors' Contributions:}

CS and NJ contributed to the conception or design of the work.

CS, NJ, MA and VF contributed to the acquisition, analysis, or interpretation of data for the work.

CS and NJ drafted the manuscript.

CS and NJ critically revised the manuscript.

All authors gave final approval and agree to be accountable for all aspects of work ensuring integrity and accuracy.

\section{References:}

Figure Legends:

\section{Figure 1. Hemodynamic waveforms obtained during the RHC}

A, Right Atrium Waveform. B, Right Ventricular Base Waveform. C, Right Ventricular Outflow Tract Waveform. D, Pulmonary Artery Waveform. E, Pulmonary Capillary Wedge Waveform.

Figure 2. TTE views demonstrating the RV moderator band and a view of the dilated right atrium

A, Parasternal short axis view demonstrating prominent $R V$ moderator band (yellow arrow). B, Apical view demonstrating prominent $R V$ moderator band (yellow arrow). C, Apical view demonstrating moderate right 
atrium enlargement $\left(19.5 \mathrm{~cm}^{2}\right)$.
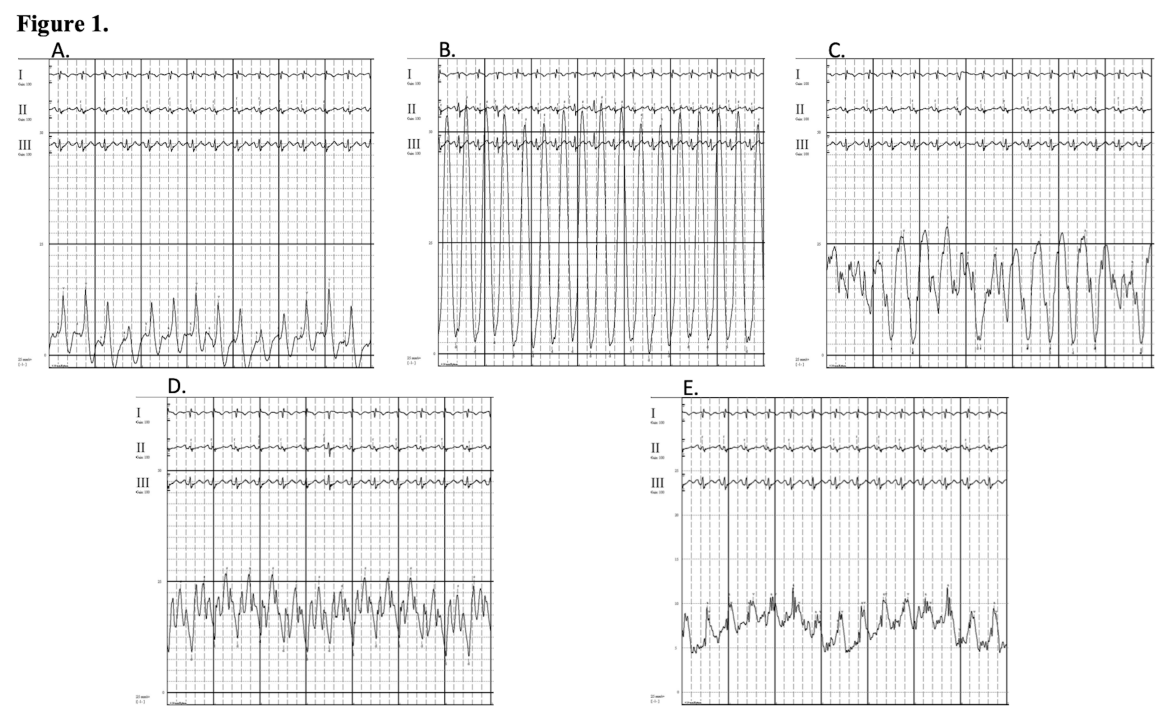

Figure 2.

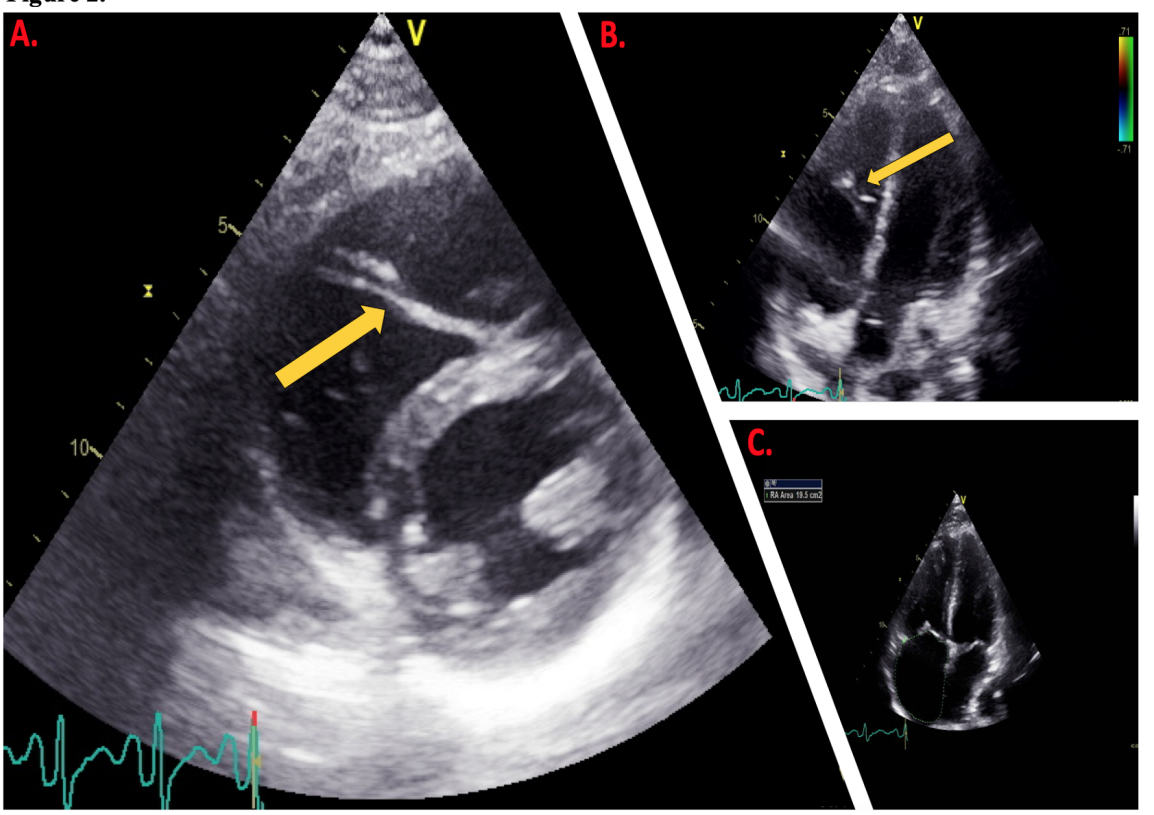

